Research Paper

\title{
Expression patterns for nicotinic acetylcholine receptor subunit genes in smoking-related lung cancers
}

\author{
Anna Bordas ${ }^{1,5, *}$, José Luis Cedillo ${ }^{1,5, *}$, Francisco Arnalich ${ }^{2,5, \#}$, Isabel Esteban- \\ Rodriguez ${ }^{3,5}$, Laura Guerra-Pastrián ${ }^{3,5}$, Javier de Castro ${ }^{4,5}$, Carolina Martín- \\ Sánchez ${ }^{1,5}$, Gema Atienza ${ }^{1,5}$, Carmen Fernández-Capitan ${ }^{2,5}$, Juan José Rios ${ }^{2,5}$ and \\ Carmen Montiel ${ }^{1,5, \#}$ \\ ${ }^{1}$ Departamento de Farmacología y Terapéutica, Facultad de Medicina, Universidad Autónoma de Madrid, Spain \\ ${ }^{2}$ Servicios de Medicina Interna, Hospital Universitario La Paz, Madrid, Spain \\ ${ }^{3}$ Anatomía Patológica, Hospital Universitario La Paz, Madrid, Spain \\ ${ }^{4}$ Oncología Médica, Hospital Universitario La Paz, Madrid, Spain \\ ${ }^{5}$ Instituto de Investigaciones Sanitarias IdiPAZ, Madrid, Spain \\ *Co-first authors, These authors contributed equally to this work \\ \#These authors have contributed equally to the direction of the work \\ Correspondence to: Carmen Montiel, email: carmen.montiel@uam.es \\ Francisco Arnalich, email: farnalich@salud.madrid.org, francisco.arnalich@uam.es \\ Keywords: $n A C h R s, N S C L C$, squamous cell carcinoma of the lung, lung adenocarcinoma, tobacco
}

Received: April 07, 2017

Accepted: June 17, 2017

Published: July 04, 2017

Copyright: Bordas et al. This is an open-access article distributed under the terms of the Creative Commons Attribution License 3.0 (CC BY 3.0), which permits unrestricted use, distribution, and reproduction in any medium, provided the original author and source are credited.

\section{ABSTRACT}

Cigarette smoking is associated with increased risk for all histologic types of lung cancer, but why the strength of this association is stronger for squamous cell carcinoma than adenocarcinoma of the lung (SQC-L, ADC-L) is not fully understood. Because nicotine and tobacco-specific nitrosamines contribute to carcinogenesis by activating nicotinic acetylcholine receptors ( $\mathrm{nAChRs)}$ on lung tumors and epithelial cells, we investigated whether differential expression of nAChR subtypes in these tumors could explain their different association with smoking. Expression of $\mathrm{nAChR}$ subunit genes in paired tumor and non-tumor lung specimens from 40 SQC-L and 38 ADC-L patients was analyzed by quantitative PCR. Compared to normal lung, both tumors share: i) transcriptional dysregulation of CHRNA3/CHRNA5/CHRNB4 (a3, a5, $\beta 4$ subunits) at the chromosomal locus that predisposes to lung cancer; and ii) decreased expression of CHRFAM7A (dupa7 subunit); this last subunit negatively modulates a7-nAChR activity in oocytes. In contrast, CHRNA7 (a7 subunit) expression was increased in SQC-L, particularly in smokers and non-survivors, while CHRNA4 (a4 subunit) expression was decreased in ADC-L. Thus, over-representation of cancer-stimulating a7-nAChR in SQC-L, also potentiated by smoking, and underrepresentation of cancer-inhibiting a4ß2-nAChR in ADC-L could explain the different tobacco influences on the tumorigenic process in each cancer type.

\section{INTRODUCTION}

Lung cancer is the leading cause of cancer-related deaths worldwide [1]. There are two major categories of lung cancer, non-small cell lung carcinoma (NSCLC) and small-cell lung carcinoma (SCLC), respectively accounting for $75-85 \%$ and $15-25 \%$ of all lung cancer cases. Lung adenocarcinoma (ADC-L) and squamous cell carcinoma of the lung (SQC-L) are the two major histologic types of NSCLC, which also includes large-cell lung carcinoma (LCLC) as a minor type.

Numerous epidemiologic and laboratory-based studies have confirmed that cigarette smoking is associated with increased risk for all major histological 
types of lung cancer, which is understandable given that tobacco contains more than 70 known carcinogens [2]. However, it is surprising how much the strength of the above association varies between the different histological tumor types, being stronger with SCLC and SQC-L than LCLC and ADC-L [3]. One of the hypotheses to justify the variation in the risk associated with smoking for one or the other tumor type, is related to the location and moreor-less intimate exposure of the tumor to inhaled tobacco smoke and its carcinogenic components. This hypothesis would explain why the more peripheral tumors (ADC-L and LCLC) show weaker associations with smoking than the more central tumors (SQC-L or SCLC) [4]. However, the above proposal does not exclude a second, so far never-explored possibility that would imply tumordependent differential expression of target receptors for the carcinogenic components of tobacco.

Tobacco smoke contains multiple classes of carcinogens, including polycyclic aromatic hydrocarbons and the nicotine-derived nitrosamines 4-(methylnitrosamino)-1-(3-pyrydyl)-1-butanone (NNK) and N-nitrosonornicotine (NNN). By inducing the formation of DNA adducts and causing mutations in vital cancer suppressor genes (i.e. $R b, p 53$ and $K$-Ras), these carcinogens eventually initiate carcinogenesis [5]. At the same time, the addictive components of tobacco, nicotine and its carcinogenic derivatives NNK and NNN, also significantly contribute to the oncogenic process after binding and activating cell surface nicotinic acetylcholine receptors (nAChRs) expressed in neuronal and nonneuronal cells, including the cancer cells themselves [6]. Stimulating the receptors in the cancer cells leads to downstream activation of multiple signaling cascades that promote cancer cell survival, proliferation, angiogenesis, migration and metastasis in a tumor-specific manner [7-10]. The nAChRs are complex structures composed of five transmembrane subunits arranged around a central ion pore [11]. To date, several alpha $(\alpha 3-\alpha 7, \alpha 9)$ and beta ( $\beta 2$ and $\beta 4)$ nAChR subunits have been identified in primary lung tumors and human lung cancer cell lines [see Ref. 12 and references therein]. Accordingly, it is likely that the above tumors express homomeric $\alpha 7$ - and $\alpha 9$ nAChRs (composed of five identical $\alpha 7$ or $\alpha 9$ subunits) and heteromeric $\alpha 3 \beta 4 \alpha 5-, \alpha 3 \beta 2 \alpha 5$ - and $\alpha 4 \beta 2-n A C h R s$ (constituted by different combinations of $\alpha$ and $\beta$ subunits).

The different subunit compositions of $\mathrm{nAChR}$ determine its functional and pharmacological characteristics as well as its pathophysiological role. In relation to this last aspect, $\alpha 9-n A C h R s$ are known to play an important role in human breast cancer progression [13], while $\alpha 7-n A C h R$ is the main subtype responsible for the nicotine-mediated proliferative, pro-angiogenic and prometastatic effects in human NSCLC [14-17]. In contrast to the $\alpha 7$-nAChR-mediated stimulatory effects on cancer growth, heteromeric $\alpha 4 \beta 2-n A C h R$ seems to be one of the major inhibitors of cancer development and progression through its release of GABA, which blocks the cancer- stimulating effects of $\beta$-adrenergic receptors by inhibiting cAMP [18]. It is interesting to note that chronic exposure to nicotine, and its carcinogenic derivative NNK, increases cancer-stimulating $\alpha 7-\mathrm{nAChR}$ expression and desensitizes cancer-inhibiting $\alpha 4 \beta 2-n A C h R$ in cancer cells in the lungs and pancreas $[16,19,20]$. This means that smoking activates cancer-stimulating $\mathrm{nAChRs}$ in the cells.

Genes CHRNA3, CHRNA4, CHRNA5, CHRNA6, CHRNA7, CHRNA9, CHRNB2 and CHRNB4, located across different chromosomes, respectively encode for the $\alpha 3, \alpha 4, \alpha 5, \alpha 6, \alpha 7, \alpha 9, \beta 2$ and $\beta 4$ nAChR subunits expressed in lung cancer cells [see Ref. 12 and references therein]. Several genome-wide association studies (GWAS) have identified a susceptibility locus for human lung cancer at chromosome 15q24-25, which contains three of the above genes: CHRNA3/CHRNA5/ CHRNB4 [21-23]. The discovery of a new hybrid gene (CHRFAM7A) in the human genome, but not in that of other higher primates, is also noteworthy. The hybrid gene results from the fusion of a partial duplication of the CHRNA7 gene with the FAM7A gene [24, 25]. Moreover, the $C H R F A M 7 A$ transcript, named dup $\alpha 7 \mathrm{mRNA}$, has been identified in brain (hippocampus, cortex, corpus callosum, thalamus, putamen, caudate nucleus and cerebellum) and in the periphery (peripheral blood mononuclear cells, lymphocytes and synoviocytes) [see Ref. 26 and references therein]. Moreover, our group performed the first study on the functional role of the new dup $\alpha 7-n A C h R$ subunit heterologous expressed in Xenopus oocytes, demonstrating that it behaves as a negative endogenous regulator of $\alpha 7-n A C h R$ activity [26]. Given the prominent role of $\alpha 7-n A C h R$ in the development of lung cancer, it is feasible that dup $\alpha 7$ could act as an endogenous suppressor of tumor growth mediated by $\alpha 7-n A C h R s$, as long as this atypical subunit was expressed in the tumor. To date, there have been no data in the literature on this question.

The present study aims to determine whether differences in the expression pattern for the genes encoding $\mathrm{nAChR}$ subunits that make up target receptors for the carcinogenic components of tobacco could explain the variability of the influence of tobacco in the two major histologic types of NSCLC. To address this issue, we have used real-time quantitative polymerase chain reaction (qPCR) to examine normalized or absolute mRNA expression levels of several $\mathrm{nAChR}$ subunits in lung biopsies from 40 patients with SQC-L and 38 patients with ADC-L. Each patient provided matched specimens, one of non-tumor lung tissue and another from their lung tumor.

\section{RESULTS}

\section{Demographic and clinical characteristics of patients}

Seventy-eight NSCLC patients were included in the study and their demographic and clinical characteristics are summarized in Table 1. Patients were grouped 
according to their histologic tumor type [40 SQC-L $(51.3 \%)$ and 38 ADC-L (48.7\%)], gender (with a majority of men in both tumor types) and age ( $87 \%$ of SQC-L and $92.1 \%$ of ADC-L patients were over 60 years old). Most SQC-L patients were smokers $(87.5 \%)$ at the time of diagnosis with a history of tobacco consumption averaging at least 20 packs per year, while more than half of the ADC-L patients $(55.3 \%)$ were non-smokers or secondhand smokers. The tumor differentiation grade according to the 2015 WHO classification of lung cancer [27] and long-term outcomes are also summarized in Table 1.

\section{Expression of $\mathrm{nAChR}$ subunit gene in tumor biopsies of SQC-L and ADC-L patients}

Gene expression of nAChR subunits was analyzed in matched tumor and non-tumor lung specimens from each patient included in the study. The upper panels of Figure 1A and 1B show the gene expression for each subunit in the tumor sample normalized with respect to its non-tumor partner for both groups of patients. The results for SQC-L tumors showed a significant increase in $\alpha 5, \alpha 7$ and $\beta 4$, together with a significant decrease in $\alpha 3$, dup $\alpha 7$ and $\beta 2$ mRNA levels (Figure 1A). We also found that ADC-L and SQC-L tumors shared significant changes in the expression of some $\mathrm{nAChR}$ genes (increased $\alpha 5$ and $\beta 4$ and reduced $\alpha 3$ and dup $\alpha 7$ mRNA levels). However, differently from the latter, ADC-L tumors showed a significant reduction in $\alpha 4$ mRNA level without significant changes in the expression of $\alpha 7$ or $\beta 2$ mRNAs (Figure 1B).

Our data concerning the normalized $\alpha 7$ and dupa7 mRNA expression in tumors of SQC-L and ADC-L patients were subsequently corroborated by the absolute expression values (number of mRNA copies) of both messengers determined in paired tumor and non-tumor biopsies from the patients (Figure 1C). The results revealed that, compared to non-tumor specimens, only SQC-L tumors showed a significant increase in absolute $\alpha 7$ mRNA expression, but that both tumor types shared a significant reduction in the number of dupa7 mRNA copies. Using the absolute expression values of both messengers in the SQC-L and ADC-L tumors, we calculated the quotient of $\alpha 7$ versus dup $\alpha 7$ mRNA molecules in each histological type as a $\alpha 7 /$ dup $\alpha 7$ ratio. The results demonstrate that, in comparison to ADC-L, SQC-L tumors contain a significantly higher proportion of $\alpha 7$ than dup $\alpha 7$ mRNA molecules (Figure 1D).

\section{Frequency distribution and correlation analysis of $\alpha 7$ expression versus expression of each of the remaining $\mathrm{nAChR}$ subunits in SQC-L and ADC-L tumors}

To obtain complementary information for that provided by Figure 1 in the groups of SQC-L and ADC-L patients, we next analyzed the frequency distribution for two categorical variables ( $\alpha 7$ versus another $\mathrm{nAChR}$ subunit), each with two categories (higher or lower mRNA expression in the tumor compared to its paired non-tumor sample) in each group. The $\alpha 7$ subunit was chosen as the fixed variable for comparison against each of the remaining variables (another nAChR subunit) because the $\alpha 7-n A C h R$ subtype is the most directly involved in tobacco-induced tumorigenesis. With the qualitative data from above, we produced a contingency table for each tumor type that was later transformed into the corresponding "stacked bar chart" (Figure 2) to facilitate visual comparison between categories (see the insert with the colored categories to the right of the figure). The numbers inside the stacked bars correspond to the percentage of patients in each segment.

The results in SQC-L patients show a statistically significant relationship between $\alpha 7 \mathrm{mRNA}$ expression and expression of dup $\alpha 7, \alpha 3, \alpha 5, \beta 2$ or $\beta 4$ mRNA (panel A). In addition, we found that the most frequent distribution in these patients was the one with increased expression of $\alpha 7$ mRNA in their tumors, which was accompanied by a decreased expression of dup $\alpha 7$ ( $43 \%$ of the patients, purple), $\alpha 3$ (44\%, purple), $\beta 2$ (44\%, purple), or increased expression of $\alpha 5$ (54\%, orange) and $\beta 4$ (70\%, orange). It should be noted that tumor levels of dup $\alpha 7, \alpha 3$ and $\beta 2$ mRNAs are decreased and those of $\alpha 5$ and $\beta 4$ increased in most SQC-L patients $(73 \%, 75 \%, 75 \%, 64 \%$ and $89 \%$, respectively).

Figure $2 \mathrm{~B}$ shows that for patients with ADC-L, the frequency distribution for each pair of variables differs from that found in SQC-L patients. Therefore, the most common distribution in ADC-L patients corresponds to that with diminished expression of $\alpha 7$ and the remaining related variables [dup $\alpha 7$ (41\%), $\alpha 3(42 \%), \alpha 9(38 \%)$ and $\beta 2(42 \%)=$ green], in their tumors. It is also noteworthy that, regardless of their $\alpha 7$ expression, most ADC-L patients showed reduced tumor expression of dupa7, $\alpha 3, \alpha 9$ and $\beta 2(70 \%, 68 \%, 62 \%$ and $74 \%$, respectively). The statistical analysis of our data does not detect an association between $\alpha 7$ and $\alpha 4$ expression in ADC-L tumors. However, and curiously, most ADC-L patients (84\%) present a down-regulation of $\alpha 4$ subunit gene expression that is accompanied by either up-regulation (in $44 \%$ ) or down-regulation (in $40 \%$ ) of $\alpha 7$ expression.

Our results also reveal the significant correlation between $\alpha 7$ mRNA expression levels and the level of expression of other nAChR subunits (dup $\alpha 7, \alpha 3$ and $\beta 2$ ) in both tumor subtypes (Table 2). Moreover, $\alpha 7$ expression levels also correlate with those of $\alpha 5$ mRNA in SQC-L, and with those of $\alpha 4, \alpha 9$ and $\beta 4$ mRNAs in ADC-L tumors.

\section{Association of smoking, tumor differentiation grade, and gender with the expression levels of several $\mathrm{nAChR}$ subunit genes in tumor biopsies from SQC-L and ADC-L patients}

SQC-L is the type of NSCLC most strongly associated with cigarette smoking, while ADC-L, as 
Table 1: Demographic and clinical characteristics of NSCLC patients

\begin{tabular}{lcc}
\hline Histologic type & SQC-L & ADC-L $^{\mathrm{a}}$ \\
\hline Number of tumor biopsies & $40(51.3 \%)$ & $38(48.7 \%)$ \\
Gender & & \\
$\quad$ Male & $32(80 \%)$ & $25(65.8 \%)$ \\
$\quad$ Female & $8(20 \%)$ & $13(34.2 \%)$ \\
Age & & \\
$\quad<60$ & $5(12.5 \%)$ & $3(7.9 \%)$ \\
$>60$ & $35(87.5 \%)$ & $35(92.1 \%)$ \\
Tobacco habit & & \\
$\quad$ Never smoker & $3(7.5 \%)$ & $11(29.0 \%)$ \\
$\quad$ Second-hand smoker & $2(5.0 \%)$ & $10(26.3 \%)$ \\
$\quad$ Smoker & $35(87.5 \%)$ & $17(44.7 \%)$ \\
Differentiation grade & & $18(47.4 \%)$ \\
$\quad$ Well/Moderate & $22(55 \%)$ & $20(52.6 \%)$ \\
$\quad$ Poor & $18(45 \%)$ & \\
Clinical outcome & & 54 months \\
$\quad$ Disease-free survival (median) & 47 months & 68.4 \\
$\quad$ Five-year survival rate (\%) & 60.0 & \\
\hline
\end{tabular}

${ }^{a} \mathrm{~A}$ non-tumor lung biopsy resected from each patient is analyzed in parallel to the paired tumor biopsy.

Table 2: Correlation between expression levels of $\alpha 7$ and other $\mathrm{nAChR}$ subunits in tumors from NSCLC patients

\begin{tabular}{|c|c|c|c|c|c|c|c|}
\hline & dupa7 & $\alpha 3$ & $\alpha 4$ & $\alpha 5$ & $\alpha 9$ & $\beta 2$ & $\beta 4$ \\
\hline SQC-L $\rightarrow \alpha 7$ & $\begin{array}{c}0.395 \\
(p=0.012)\end{array}$ & $\begin{array}{c}0.537 \\
(p=0.0001)\end{array}$ & $\begin{array}{c}-0.221 \\
(n s)\end{array}$ & $\begin{array}{c}0.506 \\
(p=0.001)\end{array}$ & $\begin{array}{c}0.302 \\
(n s)\end{array}$ & $\begin{array}{c}0.438 \\
(p=0.005)\end{array}$ & $\begin{array}{c}0.281 \\
(n s)\end{array}$ \\
\hline ADC-L $\rightarrow \alpha 7$ & $\begin{array}{c}0.548 \\
(p=0.001)\end{array}$ & $\begin{array}{c}0.676 \\
(p=0.0001)\end{array}$ & $\begin{array}{c}0.475 \\
(p=0.008)\end{array}$ & $\begin{array}{c}0.246 \\
(n s)\end{array}$ & $\begin{array}{c}0.636 \\
(p=0.0001)\end{array}$ & $\begin{array}{c}0.685 \\
(p=0.0001)\end{array}$ & $\begin{array}{c}0.387 \\
(p=0.031)\end{array}$ \\
\hline
\end{tabular}

Values represent the Spearman correlation coefficients. The statistical significance is shown in parentheses, ns $=$ not significant.

well as being the most common lung cancer in "neversmokers", is the one that shows the weakest association. Thus, we investigated whether tobacco exposure modified the expression profile of the nAChR subunit mRNAs in the tumors differently, depending on their histologic type. Figure 3A shows only those mRNAs whose normalized expression in SQC-L tumors appears to have been significantly affected by tobacco. Note the higher expression of all the affected nAChR subunits $(\alpha 7$, dup $\alpha 7, \alpha 5$ and $\alpha 9$ ) in smokers compared to nonsmokers, indicating that tobacco components up-regulate the expression of a few nAChR subunit genes in this histologic type. In contrast, none of the nAChR subunit mRNAs tested in patients with ADC-L showed changes in their expression level that could be associated to tobacco use (data not shown).

We also explored whether the degree of tumor differentiation in either histologic type could be associated to changes in the expression patterns of the nAChR subunit mRNAs. Figure 3B shows that only two subunits $(\alpha 7$ and $\alpha 5)$ showed significantly increased expression in SQC-L tumors with a poor prognosis. However, the degree of tumor differentiation did not alter the gene expression of any of the nAChR subunits assayed in ADC-L patients (data not shown). No significant gender-related expression differences were found for any of the nAChR subunit genes tested in either of the two tumor types.

\section{Gene expression pattern of different $\mathrm{nAChR}$ subunits in tumors of NSCLC patients and their relationship with survival}

Next, we investigated possible differences in the expression pattern of nAChR subunit mRNAs between patients with SQC-L or ADC-L who survived or died within 5 years after surgery. Among all the subunits analyzed, 
only $\alpha 7$ mRNA levels in SQC-L tumors showed significant differences between survivors and non-survivors. Thus, SQC-L patients who died presented significantly higher tumor $\alpha 7$ mRNA levels than those who survived. In contrast, none of the $\mathrm{nAChR}$ subunit mRNAs, including $\alpha 7$, showed any difference in expression level between ADC-L survivors and non-survivors (Figure 4, right).

\section{DISCUSSION}

This study reveals, for the first time, the existence of differences and similarities in the expression pattern of $\mathrm{nAChR}$ subunit genes between lung tumor biopsies from patients with SQC-L or ADC-L. Compared with their paired non-tumorous lung specimens, the most
A

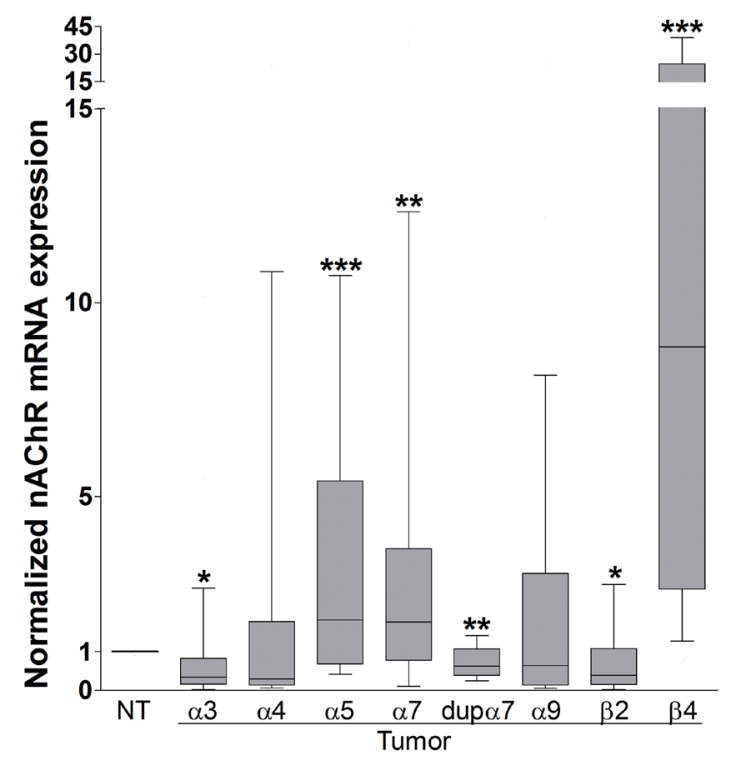

B

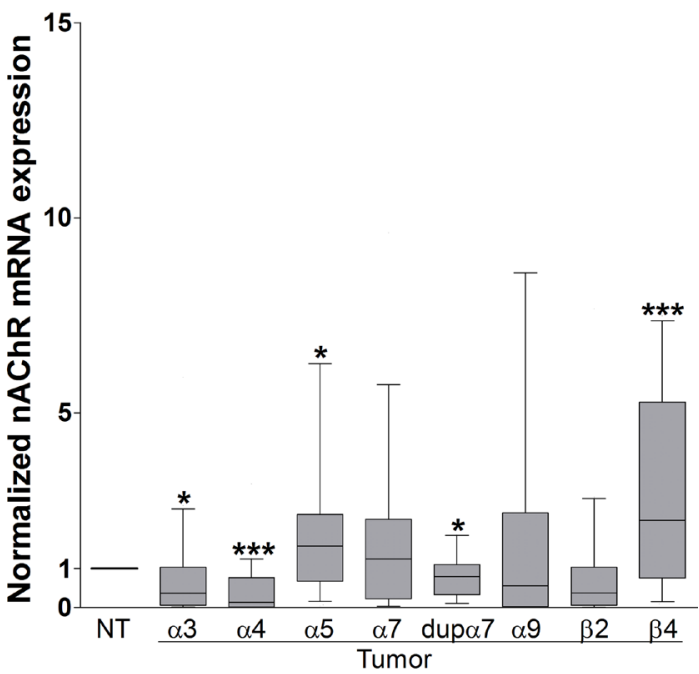

C

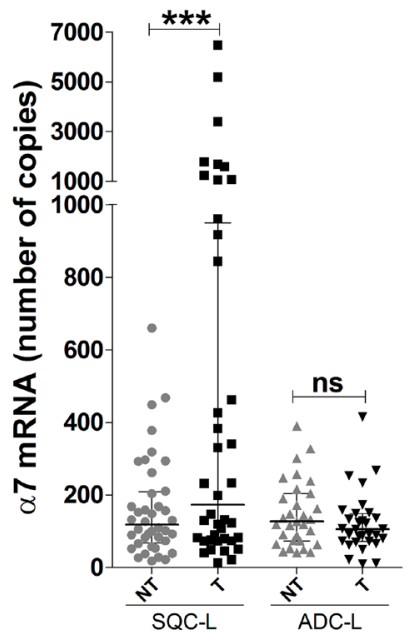

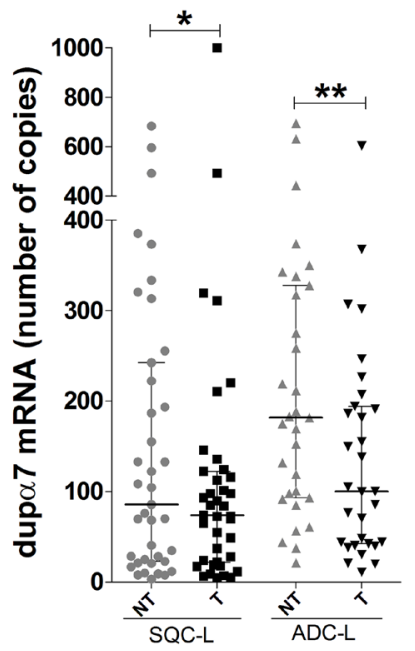

ADC-L

D

Figure 1: Expression analysis of different $n A C h R$ subunit genes in lung tumor biopsies from NSCLC patients. Expression of each gene was determined by quantitative PCR in 40 patients with SCQ-L (A) and 38 patients with ADC-L (B). Each value, obtained in triplicate and representing an average of three separate determinations, was normalized with respect to the expression of the same gene in the corresponding paired non-tumoral (NT) sample from the same patient (referred to as 1). Data are represented as a box-andwhisker plot; the line within each box shows the median expression of each subunit, upper and lower edges of the box represent the 75th and 25th percentile, and the ends of the whiskers the 90th and 10th percentile, respectively. The Wilcoxon test for paired samples was used for data analysis. ${ }^{*} p<0.05,{ }^{*} p<0.01$ and ${ }^{* * *} p<0.001$ compared to the paired non-tumor sample. (C) Dot plots representing the absolute expression number of mRNA copies for the $\alpha 7$ and dup $\alpha 7$ expressed in paired non-tumor (NT) and tumor (T) lung biopsies of 40 patients with SQC-L and 33 patients with ADC-L; panels show the median and interquartile ranges for each gene analyzed. (D) The $\alpha 7 /$ dup $\alpha 7$ ratio was calculated on the basis of the number of copies of both transcripts expressed in the tumor biopsies of the patients. Wilcoxon (C) and Mann-Whitney (D) tests were used for data analysis. ${ }^{*} p<0.05 ; * p<0.01 ; * * * p 0.001$, ns $=$ not significant. 
substantial differences are the up-regulation of $C H R N A 7$ expression in SQC-L, particularly in smokers and nonsurvivors, and the down-regulation of CHRNA4 in ADC-L. Our study also provides novel data on negative regulation of CHRFAM7A expression in SQC-L and ADC-L tumors; this gene encodes the new dupa7 nAChR subunit. Both types of tumors also share a transcriptional dysregulation of the three $\mathrm{nAChR}$ subunit genes grouped at the chromosomal locus $(15 \mathrm{q} 25.1)$ that predisposes to lung cancer; gene expression was reduced (CHRNA3) or increased (CHRNA5 and CHRNB4). Interestingly, in addition to $C H R N A 7$, only a few other nAChR subunit genes modified their expression due to the effect of tobacco (CHRFAM7A, CHRNA5, CHRNA9) or to the
B

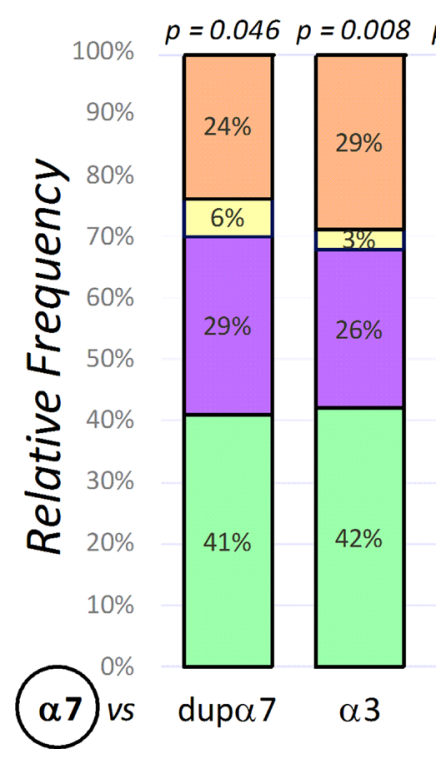

A

SQC-L
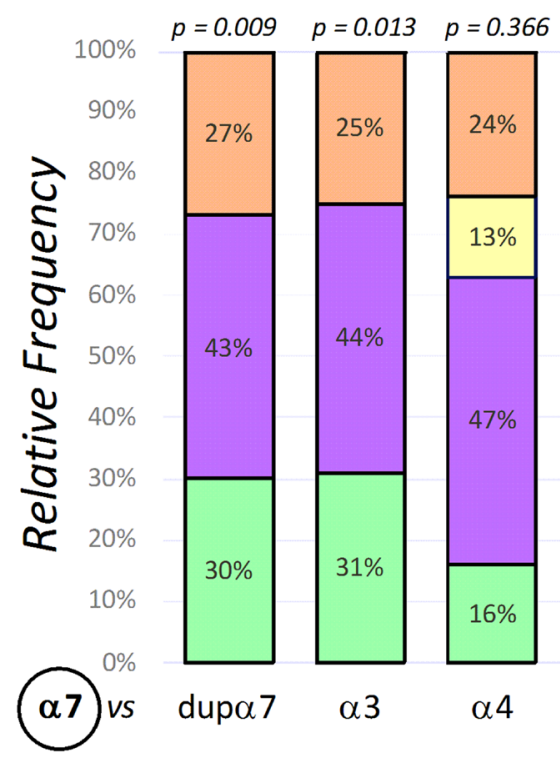

$\alpha 4$

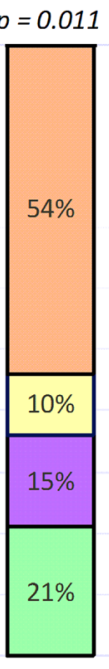

$\alpha 5$

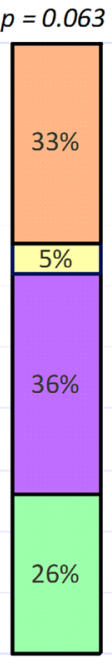

$\alpha 9$

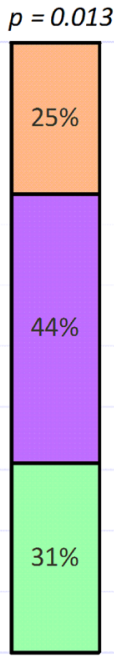

$\beta 2$

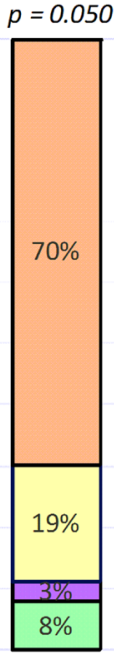

$8 \%$

$\beta 4$

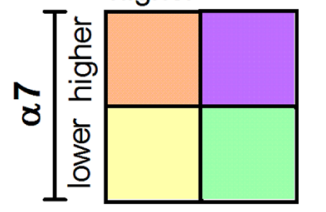

ADC-L
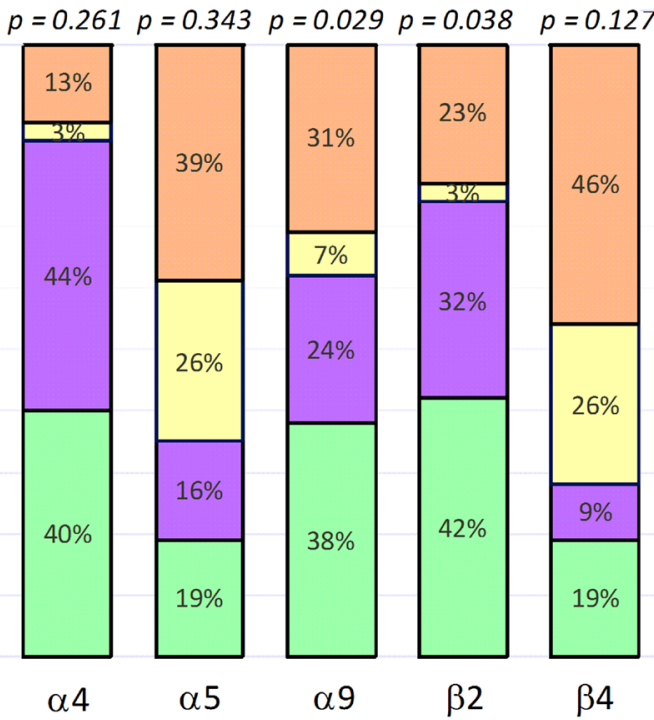

Figure 2: Frequency distribution of $\alpha 7$ expression versus expression of each of the remaining $\mathrm{nAChR}$ subunits in the SQC-L and ADC-L tumors. The stacked bar charts represent the frequency distribution, in SQC-L or ADC-L tumors, for several pairs of categorical variables ( $\alpha 7$ versus another $\mathrm{nAChR}$ subunit), each with two categories (higher or lower mRNA expression in the tumor compared to its paired non-tumor sample). Insert shows the categories identified with different colors. The numbers inside the stacked bars represent the percentage of patients in each segment. Fisher's exact test was used for analysis; statistical significance is shown at the top of the bar. 
degree of tumor differentiation (CHRNA5), but these changes only happen in SQC-L tumors. Next, we discuss the most relevant results of the present study.

Lung cancer is the leading global cause of cancer deaths, with NSCLC predominating [28]. Cells from these tumors express several nAChR subtypes whose activation by tobacco components (i.e. nicotine and its carcinogenic derivatives nitrosamines) regulates cell proliferation and apoptotic evasion [see Ref. $(12,18)$ and references therein]. Based on these observations, it has been proposed that a dysregulation of expression for genes encoding nAChR subunits and/or a dysfunction of these receptors in lung tumors might be involved in cancer-related processes. Several qPCR studies performed in NSCLC cell lines or in primary tumor biopsies from NSCLC or ADC-L patients have revealed that only a few $\mathrm{nAChR}$ subunit genes undergo dysregulation during the carcinogenic process [29]. As far as we know, no study in primary tumors from NSCLC patients has yet analyzed whether its two major histologic types (SQC-L and ADC-L) differ in their nAChR subunit gene expression pattern. Our study fills this gap and incorporates two substantial improvements in experimental design compared to previous studies in primary NSCLC tumors. The first is to include sufficient SQC-L and ADC-L patients in the same study as to be able to find, under the same experimental conditions, differences in expression of $\mathrm{nAChR}$ subunit genes between the two types of tumor. Secondly, the fact that we have had access to non-tumor lung specimens for all our patients has made it possible to detect fine changes in nAChR subunit gene expression between the tumor and its paired non-tumor sample as well as between both types of tumors.

In a previous study measuring the expression of 11 nAChR subunit genes in 66 NSCLC primary tumors (54 ADC-L/6 SQC-L/5 LCLC, 1 adenosquamous) surgically resected from Chinese patients, only two of the genes evaluated showed significant expression changes in the tumor (increased CHRNB4 and decreased CHRNA4) compared to expression levels in non-tumor tissues [29]. The findings of transcriptional dysregulation of both genes in NSCLC tumors concur with our results showing a significant increase of $\beta 4$ mRNA levels in tumors from SQC-L and ADC-L patients as well as a dramatic reduction of $\alpha 4$ mRNA levels in ADC-L tumors, the major histologic tumor group included in the Lam et al. study. However, our results differ from those of their study in that we were able to observe a larger number of $\mathrm{nAChR}$ subunit genes undergoing expression changes due to the tumorigenic process. Some of these changes are dependent on the histologic tumor type (increased $\alpha 7$ and decreased $\beta 2$ mRNA levels in SQC-L tumors) while others were
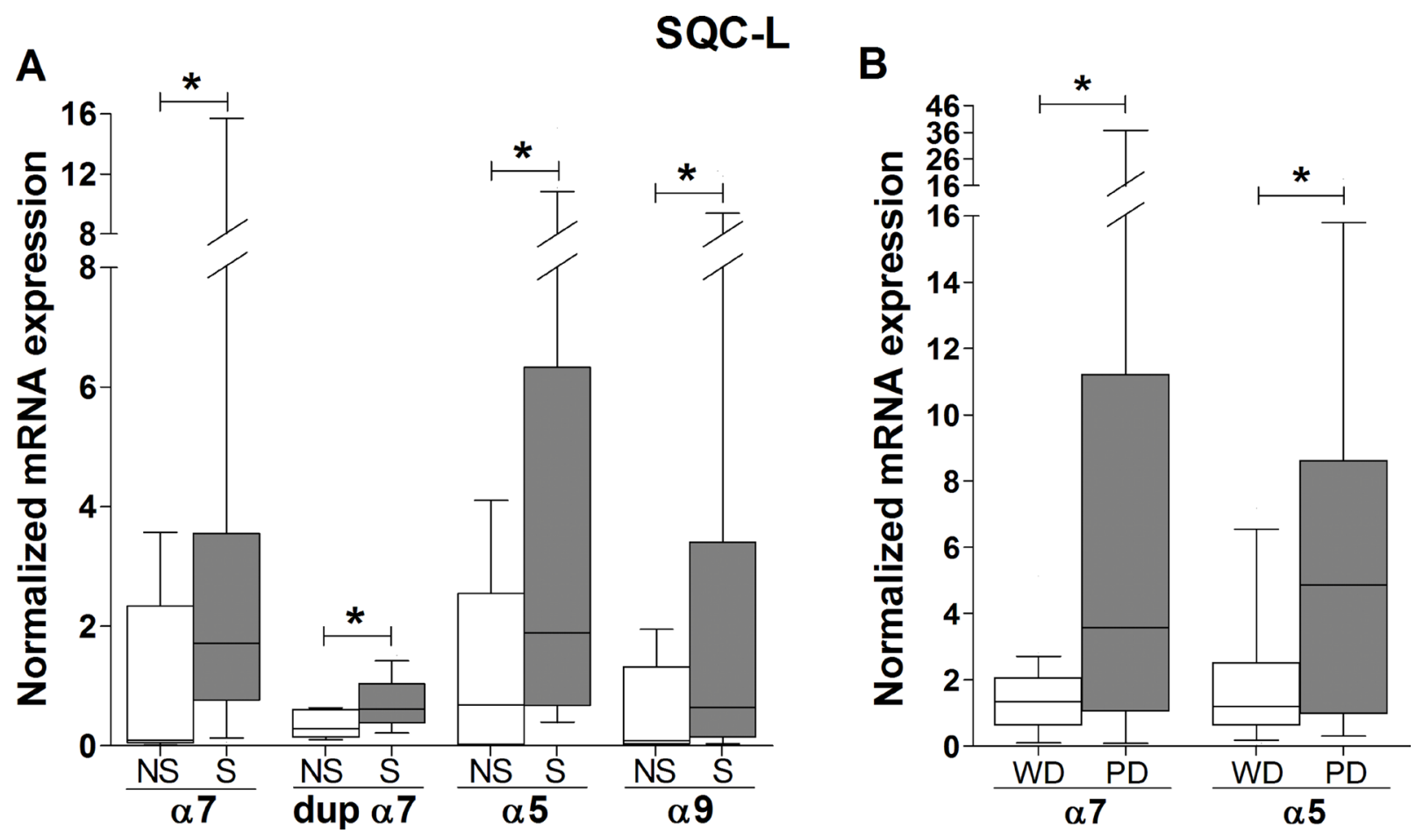

Figure 3: Association of smoking and tumor differentiation grade with the expression levels of nAChR subunit mRNAs in lung tumor biopsies from SQC-L patients. Box-plots showing the median and interquartile range of those mRNAs whose tumor expression was significantly affected by smoking (A) or tumor grade differentiation (B) in SQC-L patients [35 smokers (S) versus 5 non-smokers and second-hand smokers (NS); 22 well- or moderately-differentiated (WD) versus 18 poorly-differentiated (PD) tumors]. The data were analyzed using Student's $t$ test with unequal variance assumption (Welch's correction) (panel A) or Mann-Whitney nonparametric test (panel $\mathrm{B}$ ); ${ }^{*} p<0.05$ after comparing the indicated values. 
shared by SQC-L and ADC-L tumors (increased $\alpha 5$ and $\beta 4$ and decreased dup $\alpha 7$ and $\alpha 3$ mRNA levels). Moreover, the significant increase of $\alpha 7$ mRNA levels in SQC-L but not in ADC-L tumors found in our study when mRNA expression was determined in a normalized manner (with respect to the paired non-tumor piece), was corroborated by the data on absolute expression of $\alpha 7$ mRNA as determined in both histologic tumor types. On the other hand, changes related to $\alpha 3$ and $\alpha 5$ mRNA levels found in SQC-L and ADC-L tumors in our study concur with a previous paper describing transcriptional dysregulation for CHRNA3 and CHRNA5 in lung tumor biopsies paired with their corresponding non-tumor specimens from 21 patients with ADC-L [30].

The inability to detect expression changes for $\alpha 7$ and $\beta 2$ mRNAs in the tumors of NSCLC patients in the Lam study may be due to the small number of SQC-L patients recruited in that study (only 6 out of 66 NSCLC patients) compared to the $40 \mathrm{SQC}-\mathrm{L}$ patients included in our study. In addition, the use of the same patient>s nontumor lung sample as a calibrator in the qPCR could also have increased the sensitivity of the technique to detect changes in $\mathrm{nAChR}$ gene expression in the tumors in our

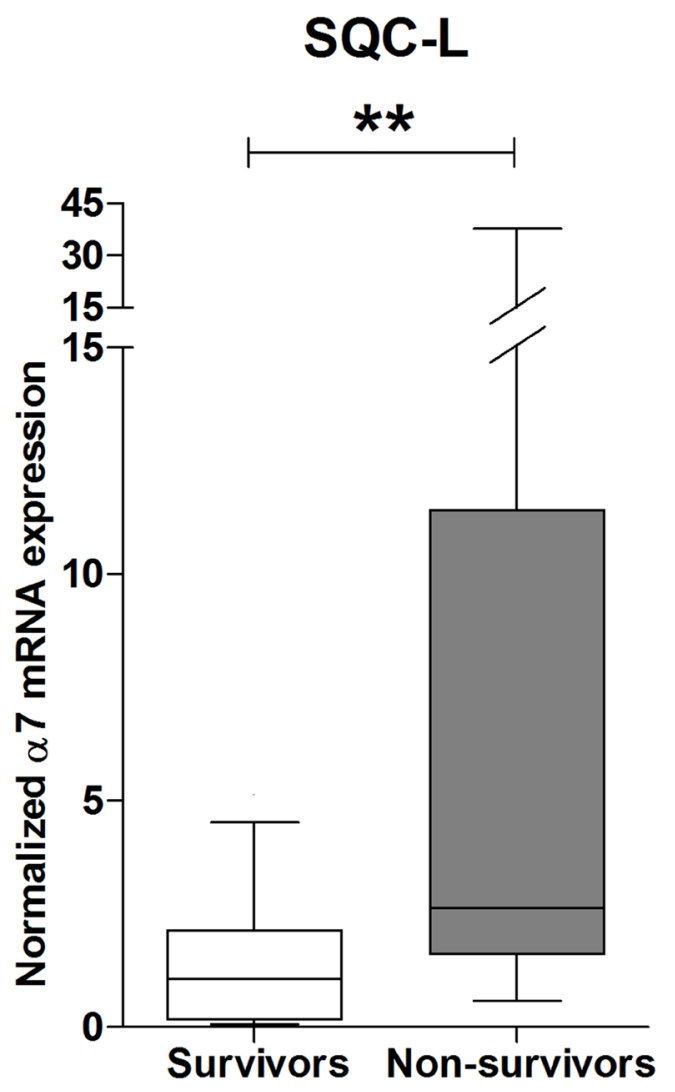

study, unlike the Lam study, which used a pool of seven non-tumor samples unrelated to the patients under study. In fact, our finding of increased $\alpha 7$ mRNA level in SQC-L tumors, especially in smokers, but not in ADC-L tumors, is consistent with previous qualitative data on $\alpha 7$ mRNA expression determined by conventional PCR in tumors from 28 SQC-L and 19 ADC-L patients [31].

Once we identified the $\mathrm{nAChR}$ subunit genes whose expression were changed by the carcinogenic process in one or another histological tumor type, the next question was whether such dysregulation could positively or negatively influence tumor growth and development. It is interesting to note that overexpression of $\alpha 7$ in SQC-L and ADC-L cell lines as compared to normal human bronchial epithelial cell lines (NHBE) has been extensively reported [29, 32]. Also, it has been shown that nicotine upregulates the expression of CHRNA7 in SQC-L and NHBE cell lines, both at the mRNA and protein levels [29, 32, 33]. Moreover, nicotine induces proliferative, survival and angiogenic effects mediated by the activation of $\alpha 7-n A C h R s$ in both types of cell lines [33-35]. In light of all of the foregoing, our results indicate that $\alpha 7-n A C h R$ would play a prominent role in the oncogenic

\section{ADC-L}

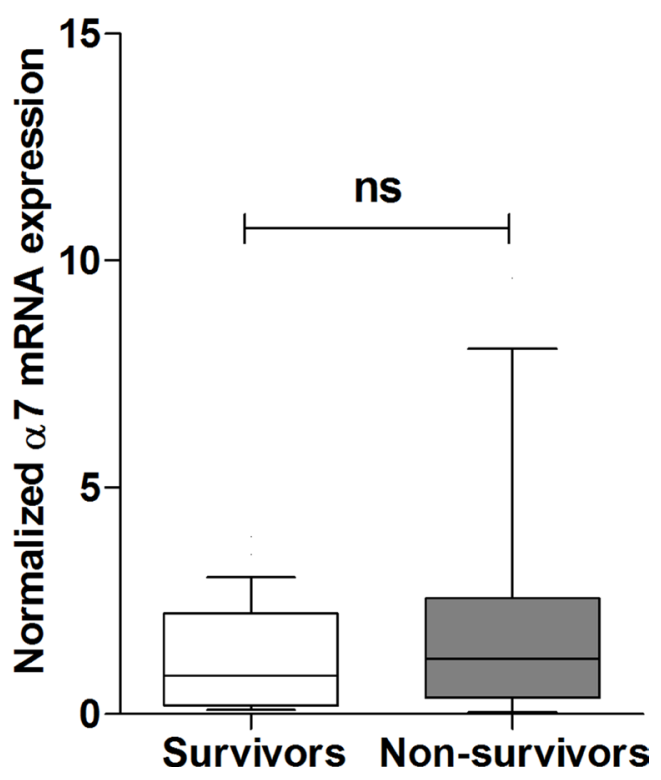

Figure 4: High expression level of $\alpha 7$ mRNA negatively influences survival of patients with SQC-L but not those with ADC-L. Box-plots show the median and interquartile range of $\alpha 7$ mRNA levels in tumor biopsies from patients with SQC-L or ADC-L surviving or not at 5-year after surgery. Unlike the rest of the analyzed genes, the only significant difference found was associated with $\alpha 7$ gene expression in SQC-L non-survivors $(n=16)$ and survivors $(n=24)$. In contrast, no significant changes in tumor expression were observed between ADC-L non-survivors $(n=12)$ and survivors $(n=26)$ for any of the nAChR subunit gene. The data were analyzed using the Mann-Whitney non-parametric test; $* * p<0.01$ after comparing the indicated values; ns = not significant. 
process of SQC-L tumors given the significant increase of CHRNA7 expression in this type of tumor, especially in smokers or in poorly differentiated tumors. Since $\alpha 7-m R N A$ levels have been correlated to the smoking history of SQC-L patients [33] and the majority of our SQC-L patients $(87.5 \%)$ are smokers, the increased $\alpha 7$ mRNA levels in SQC-L tumors found in our study were probably associated with our patients' smoking habits.

Interestingly, it has been recently revealed that the mechanism underlying nicotine-mediated up-regulation of $\alpha 7-n A C h R s$ in human SQC-L cell lines occurs at the transcription level as the result of recruitment of Sp1GATA4 or Sp1-GATA6 complexes on the CHRNA7 promoter, thereby inducing transcription and increasing $\alpha 7$ mRNA expression in these cells [see Ref. 32 and references therein]. Since $\alpha 4 \beta 2-n A C h R$ seems to be one of the major inhibitors of cancer development [18], the significant down-regulation of CHRNA4 expression in ADC-L tumors that we have found would result in a reduction in the number of nAChRs containing the $\alpha 4$ subunit, and this may favor carcinogenesis in this histological tumor type.

Our study is the first to detect and quantify the expression of the hybrid gene encoding the new dup $\alpha 7$ nAChR subunit in NSCLC tumors. In both tumor types studied here, CHRFAM7A expression was significantly lower than in the paired non-tumor lung specimens. If the dup $\alpha 7 \mathrm{nAChR}$ subunit expressed in these tumors plays a similar role to that found in Xenopus oocytes [26], it is possible that deficient dup $\alpha 7$ subunit expression facilitates the oncogenic process mediated by $\alpha 7-n A C h R s$, especially in SQC-L where overexpression of this nAChR subtype appears to be determinant to cancer progression. Further

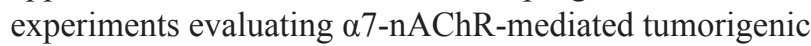
processes and their interference by dup $\alpha 7$ in NSCLC cell lines are necessary to verify the above hypothesis.

Given the association between the CHRNA3/ CHRNA5/CHRNB4 locus and the risk of lung cancer, attempts have been made to elucidate the pathophysiological role of these three genes encoding $\alpha 3, \alpha 5$ and $\beta 4 \mathrm{nAChR}$ subunits. Expression of the above genes appears to be vital for cell viability in the most aggressive type of lung cancer, SCLC. Thus, overexpression of the clustered $\mathrm{nAChR}$ genes has been reported in a human SCLC cell line, DMS-53 [36], and silencing these genes is known to reduce cell viability [12]. Our data showing overexpression of the genes encoding $\alpha 5$ and $\beta 4$ subunits in SCQ-L and ADC-L tumors agree with data obtained in SCLC cell lines. However, unlike SCLC cell lines, the CHRNA3 that encodes the $\alpha 3$ subunit is clearly downregulated in the two NSCLC types analyzed in our study. The opposite results for CHRNA3 expression in SCLC cell lines and in NSCLC tumors suggests the existence of different regulatory mechanisms for gene expression in these two categories of lung cancer. In fact, like us, other authors have found that expression of the gene encoding the $\alpha 3$ subunit is halved in lung adenocarcinoma as compared with normal lung tissue [30].

Down-regulation of CHRNA3 expression in the two tumor types in our study could be a consequence of epigenetic silencing of this gene, which is a frequent target of aberrant DNA hypermethylation [37, 38]. Overexpression of CHRNA3 by ectopic expression induces apoptotic cell death while CHRNA3 knockdown by short hairpin RNA (shRNA) abolishes the cell response to apoptosis-inducing agents in lung cancer, suggesting this gene is important to human cancer cell survival [37]. Moreover, the interaction of nicotine with nAChRs containing the $\alpha 5$ subunit promotes cell proliferation, angiogenesis and invasion through the activation of the ERK and Akt signaling pathways, upregulation of HIF-1 signaling and elevation of VEGF [39]. Thus, the downregulation of CHRNA3 expression combined with upregulation of CHRNA5 expression in our SQC-L and ADC-L tumors might result in under-representation of the $\alpha 3$-containing $\mathrm{nAChRs}$ and over-representation of the $\alpha 5$-containing nAChR subtypes on the cell surface, which could lead to a defective cell death response and again, favor the carcinogenic process.

In summary, we have observed that the number of nAChR subunit genes that undergo expression changes in the two major NSCLC histological types is higher than reported in previous studies. This observation is probably the result of the use of paired non-tumor lung samples for all patients included in the study. The separate analysis of gene expression by nAChR subunits in SQC-L and ADC-L tumors, performed for the first time in a single study, also allows us to reliably establish differences and similarities of gene expression in the two histological tumor types. Although dysregulated gene expression of certain $\mathrm{nAChR}$ subunits $(\alpha 5$ increased; $\alpha 3$ and dup $\alpha 7$ decreased) could contribute to the oncogenic process in both tumor types, the two main differences are the under-representation of the cancer-inhibiting $\alpha 4 \beta 2$ nAChR subtype in ADC-L tumors and the overexpression of the main cancer-promoting $\alpha 7-n A C h R$ subtype in SQC-L tumors. These differences could be determinant for the growth of one or the other tumor type. Our results showing higher expression levels of $\alpha 7$ mRNA in SQC-L tumors from smokers than from non-smokers, and the observation that $\alpha 4$ mRNA levels in ADC-L tumors are not affected by smoking, appear to support the stronger association of tobacco with the first tumor type than the second. Finally, our data detailing the expression pattern of the different $\mathrm{nAChR}$ subunit genes in SQC-L and ADC-L may enhance the understanding of tumorigenic processes in tobacco-related tumors.

\section{MATERIALS AND METHODS}

\section{Patients and lung samples}

Lung specimens from 78 patients diagnosed with NSCLC (40 SQC-L and 38 ADC-L) were obtained 
Table 3: List of primers used to amplify the nAChR subunit genes

\begin{tabular}{|c|c|c|c|c|c|}
\hline Subunit (reference gene) & Sequences $\left(5^{\prime}-3^{\prime}\right)$ & $\begin{array}{l}\text { Amplicon } \\
\text { size (bp) }\end{array}$ & $\begin{array}{l}\mathrm{Tm} \\
\left({ }^{\circ} \mathrm{C}\right)\end{array}$ & $\begin{array}{c}\text { GC content } \\
(\%)\end{array}$ & Reference \\
\hline$\alpha 3$ (CHRNA3) & $\begin{array}{l}\text { CAGAGTCCAAAGGCTGCAAG } \\
\text { AGAGAGGGACAGCACAGCAT }\end{array}$ & 149 & $\begin{array}{l}65 \\
64\end{array}$ & $\begin{array}{l}55 \\
55\end{array}$ & Lips et al. 2005 \\
\hline$\alpha 4(C H R N A 4)$ & $\begin{array}{l}\text { GTGGATGAGAAGAACCAGATGATG } \\
\text { CAGCGCAGCTTGTAGTCGTG }\end{array}$ & 74 & $\begin{array}{l}59 \\
62\end{array}$ & $\begin{array}{l}46 \\
60\end{array}$ & Lam et al. 2007 \\
\hline$\alpha 5(C H R N A 5)$ & $\begin{array}{l}\text { CTTCACACGCTTCCCAAACT } \\
\text { CTTCAACAACCTCACGGACA }\end{array}$ & 187 & $\begin{array}{l}64 \\
64\end{array}$ & $\begin{array}{l}50 \\
50\end{array}$ & Lips et al. 2005 \\
\hline$\alpha 7(C H R N A 7)$ & $\begin{array}{l}\text { GCTGCAAATGTCTTGGACAGAT } \\
\text { AACAGTCTTCACCCCTGGATAT }\end{array}$ & 70 & $\begin{array}{l}59 \\
59\end{array}$ & $\begin{array}{l}45 \\
45\end{array}$ & Cedillo et al. 2014 \\
\hline $\operatorname{dup\alpha } 7(C H R F A M 7 A)$ & $\begin{array}{l}\text { CAATTGCTAATCCAGCATTTGTGG } \\
\text { CCCAGAAGAATTCACCAACACG }\end{array}$ & 102 & $\begin{array}{l}59 \\
60\end{array}$ & $\begin{array}{l}42 \\
50\end{array}$ & Present paper \\
\hline$\alpha 9(C H R N A 9)$ & $\begin{array}{l}\text { AAAGATGAACTGGTCCCATTCCT } \\
\text { AAGGTCATTAAACAACTTCTGAGCATAT }\end{array}$ & 118 & $\begin{array}{l}60 \\
60\end{array}$ & $\begin{array}{l}43 \\
32\end{array}$ & Lam et al. 2007 \\
\hline$\beta 2(C H R N B 2)$ & $\begin{array}{l}\text { CTGGATCCTTCCCGCTACAAC } \\
\text { TGGGTCAGCCAGACATTGGT }\end{array}$ & 146 & $\begin{array}{l}60 \\
61\end{array}$ & $\begin{array}{l}57 \\
55\end{array}$ & Lam et al. 2007 \\
\hline$\beta 4(C H R N B 4)$ & $\begin{array}{l}\text { TCACAGCTCATCTCCATCAAGCT } \\
\text { CCTGTTTCAGCCAGACATTGGT }\end{array}$ & 100 & $\begin{array}{l}62 \\
61\end{array}$ & $\begin{array}{l}48 \\
50\end{array}$ & Lam et al. 2007 \\
\hline
\end{tabular}

from the IdiPAZ Biobank at the Medical University Hospital La Paz, Madrid. Patients underwent lung tumor resection and systematic lymph node dissection between January 2007 and June 2010. The specimens of tumor lung biopsy from each patient, along with their corresponding paired non-tumor paired lung tissue, were respectively obtained from the center of the tumor and from a non-infiltrated area of the lung distant from the tumor, and were confirmed by histopathological evaluation (7th edition of the TNM Classification for Lung Cancer). Optimal cutting temperature (OCT)embedded tissue specimens were snap-frozen in liquid nitrogen and stored at $-80^{\circ} \mathrm{C}$ until RNA extraction, and then categorized according to clinical information by the Hospital Biobank. After being duly informed, all patients had given written permission for their samples to be used in research. The Institutional Ethics Committee of our hospital approved the study. Histological grading of NSCLC types was based on the revised World Health Organization (WHO) classification of lung tumors [27]. Lepidic-predominant adenocarcinomas were classified as well-differentiated (G1), papillary-predominant and acinar-predominant adenocarcinomas were classified as moderately-differentiated (G2), while soli-predominant and micropapillary-predominant adenocarcinomas were classified as poorly-differentiated (G3). Squamous cell carcinomas with extensive keratinization are welldifferentiated, while those with little to no keratinization were classified as poorly-differentiated. The complete follow-up information was recorded; the last date of follow-up for survivors occurred at the end of July 2015. The five-year survival rate was defined as the percentage of patients surviving five years after surgery. Disease-free survival was defined as the median time (months) between surgery and lung cancer recurrence. The interval between follow-up examinations was every 3 months for the first 2 years and every 4 months for the following 3 years.

\section{Reverse transcription of RNA and quantitative real-time PCR (qPCR)}

Prior to RNA extraction, the tumor or non-tumoral nature of all lung specimens included in the study was histopathologically confirmed. Techniques for RNA extraction from tissues or cells, as well as gene expression assay by qPCR from reverse-transcribed RNA using SYBR green-based assays (Bio-Rad, Hercules, CA) and the ABI Prism 7500 Sequence Detector (Applied Biosystems, Foster City, CA), have been described elsewhere $[26,40]$. Briefly, total RNA was extracted from lung biopsy specimens (60 to $200 \mathrm{mg}$ ) using the Qiagen RNeasy Mini Kit and cDNAs were generated by reverse transcription of total RNA (300 ng) using Taqman Reverse Transcription Reagents (Life Technologies) according to the manufacturer's instructions. The set of primers listed in Table 3 and the following cycling conditions $\left(95^{\circ} \mathrm{C}\right.$ for $30 \mathrm{~s}$, followed by 40 cycles at $95^{\circ} \mathrm{C}$ for $5 \mathrm{~s}$ and $60^{\circ} \mathrm{C}$ for $30 \mathrm{~s}$ ) were used for PCR amplification of nAChR genes. The next pair of primers [5'-TGATCAAGGGAAAGATGACCA-3', 5'-AACCCTCTTGCAA-TCGAAAA-3'] was employed to amplify the human D esterase gene (ESD) which was selected as an endogenous control for the PCR reaction since it is well-established that this gene is one of the most appropriate to study the gene expression profile in non-small cell lung cancer [41, 42]. All determinations were performed in triplicate and paired tumor and non- 
tumor biopsy specimens were always analyzed in the same analytical run to avoid between-run variations. Reaction conditions were validated separately for each pair of primers, with single peak dissociation curves produced in each reaction run. The relative changes in mRNA expression in tumor biopsies for all $\mathrm{nAChR}$ subunits were assessed by the $2^{-\Delta \Delta \mathrm{Ct}}$ method, using their corresponding non-tumor paired specimens as calibrator. The number of $\alpha 7$ and dup $\alpha 7$ mRNA copies expressed in paired tumor and non-tumor biopsies were determined using a six-point standard curve prepared with the plasmids pcDNA3.1 $\alpha 7$ and pcDNA3.1dupa7-myc-His (our own laboratory preparation) as templates and applying the following formula: number $=(\mathrm{ng} *$ number/mole $) /(\mathrm{bp} * \mathrm{ng} / \mathrm{g} * \mathrm{~g} /$ mole of bp) (cite website: http://cels.uri.edu/gsc/cndna. html).

\section{Statistical analysis}

Differences in nAChR mRNA levels between tumor and non-tumor paired specimens from NSCLC patients were assessed by the Wilcoxon test. The Mann-Whitney test was used to assess differences between SQC-L or ADC-L tumors in regard to the $\alpha 7 /$ dup $\alpha 7$ mRNA ratio. The Spearman coefficient was used to analyze the correlation between expression levels of $\alpha 7$ mRNAs and other nAChR subunit mRNAs in tumor biopsies from NSCLC patients. The relationship between two related variables $(\alpha 7 \mathrm{mRNA}$ expression $v s$ expression of each of the remaining $\mathrm{nAChR}$ subunits) in the tumor as compared to its paired non-tumor sample was analyzed by Fisher's exact test. A normal distribution was assumed for groups with more than 30 patients (Central Limit Theorem) to compare expression for different $\mathrm{nAChR}$ subunit genes between groups of patients with different histological stages, smoking status and clinical outcome within 5 years after surgery. To perform the above comparisons in groups smaller than 30 patients, the Kolmogorov-Smirnov test was applied to evaluate the normality of the data; Student's $t$ test with unequal variance assumption (Welch's correction) or Mann-Whitney non-parametric test were applied for normal or non-normal distribution, respectively. A $p$ value $<0.05$ was considered statistically significant. Analysis was performed with the SPSS software package (version 17.0) and Graphpad Prism 5.0.

\section{Abbreviations}

ADC-L, lung adenocarcinoma; LCLC, largecell lung carcinoma; $\mathrm{nAChR}$, neuronal nicotinic acetylcholine receptor; NNK, nicotine-derived nitrosamines 4-(methylnitrosamino)-1-(3-pyrydyl)-1-butanone; NNN, N-nitrosonornicotine; NSCLC, non-small cell lung carcinoma; qPCR, real time quantitative polymerase chain reaction; SCLC, small-cell lung carcinoma; SQC-L, squamous cell carcinoma of the lung.

\section{Authors' contributions}

Conceived and designed the experiments: $\mathrm{CM}$ and FA. Performed RNA extractions and PCR experiments: AB, JLC, GA, CMS. Selection of tumors and collection of clinical data: FA, JC, CFC and JJR. Histological diagnosis: MIE-R and LG-P. Analyzed the data: AB and JLC. Wrote the paper: CM and FA.

\section{ACKNOWLEDGMENTS}

The authors thank the following doctors from La Paz University Hospital, Madrid: Professor David Hardisson (Head of Anatomic Pathology Service) for his useful advice and assistance to bring up this research; Dr. Marta Mendiola (Anatomic Pathology Service) for her help with tissue collection; Professor Jaime Feliú (Head of Medical Oncology Service) and the IdiPAZ Tumor Bank for providing the tumor biopsies. We also thank Prof. Juan José de la Cruz (Department of Preventive Medicine and Public Health, Medical School, Universidad Autónoma de Madrid) for his advice in conducting statistical analysis.

\section{CONFLICTS OF INTEREST}

The authors have declared no conflicts of interest.

\section{FUNDING}

This study was supported by grants to C. Montiel and F. Arnalich from the Ministry of Economy, Industry and Competitiveness, Government of Spain (SAF2014-56623-R) and Foundation "Mutua Madrileña Investigación Biomédica" (FMM2011), Spain. A.B. is recipient of a fellowship (Beca FPI, Universidad Autónoma Madrid). J.L.C. and C.M.S. are recipients of fellowships (Beca FPU from Ministerio de Educación, Cultura y Deporte and Beca FPI from Ministry of Economy, Industry and Competitiveness, Government of Spain, respectively).

\section{REFERENCES}

1. Cancer Incidence and Mortality Worldwide: IARC CancerBase No. 11 http:/www.who.int/mediacentre/factsheets/fs297/en/.

2. Hecht SS. Lung carcinogenesis by tobacco smoke. Int J Cancer. 2012; 131:2724-2732. doi: 10.1002/ijc.27816.

3. Khuder SA, Mutgi AB. Effect of smoking cessation on major histologic types of lung cancer. Chest. 2001; 120: 1577-1583. doi: 10.1378/chest.120.5.1577.

4. Yang CP, Gallagher RP, Weiss NS, Band PR, Thomas DB, Russell DA. Differences in incidence rates of cancers of the 
respiratory tract by anatomic subsite and histologic type: an etiologic implication. J Natl Cancer Inst. 1989; 81:1828-1831.

5. Schaal C, Chellappan S. Nicotine-Mediated Regulation of Nicotinic Acetylcholine Receptors in Non-Small Cell Lung Adenocarcinoma by E2F1 and STAT1 Transcription Factors. PLoS One. 2016; 11:e0156451. doi: 10.1371/ journal.pone.0156451.

6. Wessler I, Kirkpatrick CJ. Acetylcholine beyond neurons: the non-neuronal cholinergic system in humans. $\mathrm{Br}$ J Pharmacol. 2008; 154:1558-1571. doi: 10.1038/ bjp.2008.185.

7. Hecht SS. Tobacco carcinogens, their biomarkers and tobacco-induced cancer. Nat Rev Cancer. 2003; 3:733-744. doi: $10.1038 / \mathrm{nrc} 1190$.

8. Dasgupta P, Rizwani W, Pillai S, Kinkade R, Kovacs M, Rastogi S, Banerjee S, Carless M, Kim E, Coppola D, Haura E, Chellappan S. Nicotine induces cell proliferation, invasion and epithelial-mesenchymal transition in a variety of human cancer cell lines. Int J Cancer. 2009; 124:36-45. doi: 10.1002/ijc.23894.

9. Grando SA. Connections of nicotine to cancer. Nat Rev Cancer. 2014; 14:419-429. doi: 10.1038/nrc3725.

10. Schaal C, Chellappan SP. Nicotine-mediated cell proliferation and tumor progression in smoking-related cancers. Mol Cancer Res. 2014; 12:14-23. doi: 10.1158/1541-7786.MCR13-0541.

11. Changeux JP. Nicotinic receptors and nicotine addiction. C R Biol. 2009; 332:421-425. doi: 10.1016/j.crvi.2009.02.005.

12. Improgo MR, Soll LG, Tapper AR, Gardner PD. Nicotinic acetylcholine receptors mediate lung cancer growth. Front Physiol. 2013; 4:251. doi: 10.3389/fphys.2013.00251.

13. Lee CH, Huang CS, Chen CS, Tu SH, Wang YJ, Chang YJ, Tam KW, Wei PL, Cheng TC, Chu JS, Chen LC, Wu CH, Ho YS. Overexpression and activation of the alpha9nicotinic receptor during tumorigenesis in human breast epithelial cells. J Natl Cancer Inst. 2010; 102:1322-1335. doi: 10.1093/jnci/djq300.

14. Singh S, Pillai S, Chellappan S. Nicotinic acetylcholine receptor signaling in tumor growth and metastasis. J Oncol. 2011; 456743. doi: 10.1155/2011/456743.

15. Pillai S, Chellappan S. Alpha7 Nicotinic Acetylcholine Receptor Subunit in Angiogenesis and Epithelial to Mesenchymal Transition. Curr Drug Targets. 2012; 13: 671-679.

16. Schuller HM. Regulatory role of the alpha7 nAChR in cancer. Curr Drug Targets. 2012; 13:680-687.

17. Zhang C, Ding XP, Zhao QN, Yang XJ, An SM, Wang H, $\mathrm{Xu} \mathrm{L}$, Zhu L, Chen HZ. Role of alpha7-nicotinic acetylcholine receptor in nicotine-induced invasion and epithelial-to-mesenchymal transition in human non-small cell lung cancer cells. Oncotarget. 2016; 7:59199-59208. doi: 10.18632/oncotarget.10498.

18. Schuller HM. Is cancer triggered by altered signalling of nicotinic acetylcholine receptors? Nat Rev Cancer. 2009; 9:195-205. doi: 10.1038/nrc2590.

19. Schuller HM, Orloff M. Tobacco-specific carcinogenic nitrosamines. Ligands for nicotinic acetylcholine receptors in human lung cancer cells. Biochem Pharmacol. 1998; 55:1377-1384. doi: 10.1016/S0006-2952(97)00651-5.

20. Russo P, Del Bufalo A, Milic M, Salinaro G, Fini M, Cesario A. Cholinergic receptors as target for cancer therapy in a systems medicine perspective. Curr Mol Med. 2014; 14:1126-1138.

21. Amos CI, Wu X, Broderick P, Gorlov IP, Gu J, Eisen T, Dong Q, Zhang Q, Gu X, Vijayakrishnan J, Sullivan K, Matakidou A, Wang Y, et al. Genome-wide association scan of tag SNPs identifies a susceptibility locus for lung cancer at 15q25.1. Nat Genet. 2008; 40:616-622. doi: 10.1038/ ng. 109.

22. Hung RJ, McKay JD, Gaborieau V, Boffetta P, Hashibe M, Zaridze D, Mukeria A, Szeszenia-Dabrowska N, Lissowska J, Rudnai P, Fabianova E, Mates D, Bencko V, et al. A susceptibility locus for lung cancer maps to nicotinic acetylcholine receptor subunit genes on $15 \mathrm{q} 25$. Nature. 2008; 452:633-637. doi: 10.1038/nature06885.

23. Thorgeirsson TE, Geller F, Sulem P, Rafnar T, Wiste A, Magnusson KP, Manolescu A, Thorleifsson G, Stefansson H, Ingason A, Stacey SN, Bergthorsson JT, Thorlacius S, et al. A variant associated with nicotine dependence, lung cancer and peripheral arterial disease. Nature. 2008; 452:638-642. doi: 10.1038/nature06846.

24. Gault J, Robinson M, Berger R, Drebing C, Logel J, Hopkins J, Moore T, Jacobs S, Meriwether J, Choi MJ, Kim EJ, Walton K, Buiting K, et al. Genomic organization and partial duplication of the human alpha7 neuronal nicotinic acetylcholine receptor gene (CHRNA7). Genomics. 1998; 52:173-185. doi: 10.1006/geno.1998.5363.

25. Locke DP, Archidiacono N, Misceo D, Cardone MF, Deschamps S, Roe B, Rocchi M, Eichler EE. Refinement of a chimpanzee pericentric inversion breakpoint to a segmental duplication cluster. Genome Biol. 2003; 4:R50. doi: $10.1186 / \mathrm{gb}-2003-4-8-\mathrm{r} 50$.

26. de Lucas-Cerrillo AM, Maldifassi MC, Arnalich F, Renart J, Atienza G, Serantes R, Cruces J, SánchezPacheco A, Andrés-Mateos E, Montiel C. Function of partially duplicated human alpha77 nicotinic receptor subunit CHRFAM7A gene: potential implications for the cholinergic anti-inflammatory response. J Biol Chem. 2011; 2861:594-606. doi: 10.1074/jbc.M110.180067.

27. Travis WD, Brambilla E, Burke AP, Marx A, Nicholson AG. WHO Classification of Tumors of the Lung, Pleura, Thymus and Heart. Lyon: International Agency for Research on Cancer. 2015.

28. Siegel RL, Miller KD, Jemal A. Cancer Statistics, 2017. CA Cancer J Clin. 2017; 67:7-30. doi: 10.3322/caac.21387.

29. Lam DC, Girard L, Ramirez R, Chau WS, Suen WS, 
Sheridan S, Tin VP, Chung LP, Wong MP, Shay JW, Gazdar AF, Lam WK, Minna JD. Expression of nicotinic acetylcholine receptor subunit genes in non-small-cell lung cancer reveals differences between smokers and nonsmokers. Cancer Res. 2007; 67:4638-4647. doi: 10.1158/0008-5472.CAN-06-4628.

30. Falvella FS, Galvan A, Frullanti E, Spinola M, Calabro E, Carbone A, Incarbone M, Santambrogio L, Pastorino U, Dragani TA. Transcription deregulation at the $15 \mathrm{q} 25$ locus in association with lung adenocarcinoma risk. Clin Cancer Res. 2009; 15:1837-1842. doi: 10.1158/1078-0432.CCR08-2107.

31. Paleari L, Catassi A, Ciarlo M, Cavalieri Z, Bruzzo C, Servent D, Cesario A, Chessa L, Cilli M, Piccardi F, Granone P, Russo P. Role of alpha7-nicotinic acetylcholine receptor in human non-small cell lung cancer proliferation. Cell Prolif. 2008; 41:936-959. doi: 10.1111/j.13652184.2008.00566.x.

32. Lau JK, Brown KC, Thornhill BA, Crabtree CM, Dom AM, Witte TR, Hardman WE, McNees CA, Stover CA, Carpenter AB, Luo H, Chen YC, Shiflett BS, et al. Inhibition of cholinergic signaling causes apoptosis in human bronchioalveolar carcinoma. Cancer Res. 2013; 73:1328-1339. doi: 10.1158/0008-5472.CAN-12-3190.

33. Brown KC, Perry HE, Lau JK, Jones DV, Pulliam JF, Thornhill BA, Crabtree CM, Luo H, Chen YC, Dasgupta P. Nicotine induces the up-regulation of the alpha7-nicotinic receptor (alpha7-nAChR) in human squamous cell lung cancer cells via the Sp1/GATA protein pathway. J Biol Chem. 2013; 288:33049-33059. doi: 10.1074/jbc. M113.501601.

34. Dasgupta P, Rastogi S, Pillai S, Ordonez-Ercan D, Morris M, Haura E, Chellappan S. Nicotine induces cell proliferation by beta-arrestin-mediated activation of Src and Rb-Raf-1 pathways. J Clin Invest. 2006; 116:2208-2217. doi: 10.1172/JCI28164.

35. Dasgupta P, Chellappan SP. Nicotine-mediated cell proliferation and angiogenesis: new twists to an old story. Cell Cycle. 2006; 5:2324-8. doi: 10.4161/cc.5.20.3366.

36. Improgo MR, Schlichting NA, Cortes RY, Zhao-Shea R, Tapper AR, Gardner PD. ASCL1 regulates the expression of the CHRNA5/A3/B4 lung cancer susceptibility locus. Mol
Cancer Res. 2010; 8:194-203. doi: 10.1158/1541-7786. MCR-09-0185.

37. Paliwal A, Vaissiere T, Krais A, Cuenin C, Cros MP, Zaridze D, Moukeria A, Boffetta P, Hainaut P, Brennan P, Herceg Z. Aberrant DNA methylation links cancer susceptibility locus $15 \mathrm{q} 25.1$ to apoptotic regulation and lung cancer. Cancer Res. 2010; 70:2779-2788. doi: 10.1158/0008-5472.CAN-09-4550.

38. Mani S, Szymanska K, Cuenin C, Zaridze D, Balassiano K, Lima SC, Matos E, Daudt A, Koifman S, Filho VW, Menezes AM, Curado MP, Ferro G, et al. DNA methylation changes associated with risk factors in tumors of the upper aerodigestive tract. Epigenetics. 2012; 7:270-277. doi: 10.4161/epi.7.3.19306.

39. Ma X, Jia Y, Zu S, Li R, Jia Y, Zhao Y, Xiao D, Dang N, Wang Y. alpha5 Nicotinic acetylcholine receptor mediates nicotine-induced HIF-1alpha and VEGF expression in non-small cell lung cancer. Toxicol Appl Pharmacol. 2014; 278:172-179. doi: 10.1016/j.taap.2014.04.023.

40. Cedillo JL, Arnalich F, Martin-Sanchez C, Quesada A, Rios JJ, Maldifassi MC, Atienza G, Renart J, FernándezCapitán C, García-Rio F, López-Collazo E, Montiel C. Usefulness of alpha7 Nicotinic Receptor Messenger RNA Levels in Peripheral Blood Mononuclear Cells as a Marker for Cholinergic Antiinflammatory Pathway Activity in Septic Patients: Results of a Pilot Study. J Infect Dis. 2014; 211:146-155. doi: 10.1093/infdis/jiu425.

41. Saviozzi S, Cordero F, Lo Iacono M, Novello S, Scagliotti GV, Calogero RA. Selection of suitable reference genes for accurate normalization of gene expression profile studies in non-small cell lung cancer. BMC Cancer. 2006;6:200. doi: 10.1186/1471-2407-6-200.

42. Gresner P, Gromadzinska J, Wasowicz W. Reference genes for gene expression studies on non-small cell lung cancer. Acta Biochim Pol. 2009; 56:307-316. 